\section{Inquiry through practice: The brief as a pedagogical method for design research}

\section{Tatiana Tavares ${ }^{\mathrm{a}}$ \\ Marcos Mortensen Steagall ${ }^{b}$}

\section{Auckland University of Technology}

Learning and teaching in areas that require high levels of creativity, like Design and Art, can differ from other educational domains and methodologies. It may consider the complexity involving emergent properties activated from the interaction between many variables, including the researchers' participation in what is researched.

Design-based research methodology provides navigation for teaching experiences where learning outcomes are forged using briefs as design experiments or a way to carry out formative research to test and refine educational principles derived from previous knowledge.

In this study, the brief operated as a pedagogical method to combine academic conventions of design research and practice. Using a learning and teaching experience with Communication Design students in Aotearoa/New Zealand, this study presents the methods applied in a paper brief that integrated social, technical, and cognitive dimensions of knowledge construction. The brief "Auckland Plan 2050: Promoting and researching a design plan for a growing city" was delivered to level seven students over twelve weeks period and employed several studio-driven activities. As a pedagogical approach, the design studio provided a space that privileged imagination, reflection-in-action over the empirical and the rational. The studio valued the learner's worldview: their geographic localities, culture, their communities and the impact of the design to a broader context. Understanding the dynamics given by these spaces created opportunities to consider design teaching methods that were collaborative, informal, generative, and supportive. The studio-driven classroom brought research and practice together, and offered social media and emerging technologies as a tool for iteration and communication processes.

The brief shed light on Social Design and started with a hypothetical research question: How do design outcomes increase awareness of a real-world problem? Using a Council's long-term plan for Auckland city, students investigate specific
Orcid number issues, and challenges communities will face and design solutions that were industry, research-driven and culturally reflecting Kaupapa Māori values. During sessions with Māori scholars, entrepreneurs, and the design community, the brief provided a discursive platform that converged the design industry, stakeholders, and academia. The reflection about this complex social, cultural, and ecological network considered Auckland's inhabitants' needs and aspirations, enlightening a social perspective to design students.

As a result, students developed award-winning cohesive design artefacts and extensive exegetical contextual analysis and documentation of the process. The outcomes branched from diverse media forms, including branding, graphic design, wayfinding, UX/UI, AR, and VR technologies. The moderation process between designers, academic staff, and stakeholders during a 3-year cycle demonstrated a successful model for integrating industry expertise and academic rigour, crafted through a paradigm oriented by practice. Surveys with students indicated a positive response associated with designing under real-world settings, which increased engagement and provided strategic platforms for iteration, dialogue, collaboration, and cultural diversity. 